\title{
Maser kinematics and infrared properties of the water fountain IRAS 18286-0959
}

\author{
Bosco H. K. Yung ${ }^{1}$, Jun-ichi Nakashima ${ }^{1}$, Hiroshi Imai ${ }^{2}$, \\ Shuji Deguchi ${ }^{3}$, Philip J. Diamond ${ }^{4,5}$ and Sun Kwok ${ }^{1}$ \\ ${ }^{1}$ Department of Physics, The University of Hong Kong, Pokfulam Rd, Hong Kong, China \\ B. H. K. Yung's email: byung@hku.hk \\ ${ }^{2}$ Graduate School of Science and Engineering, Kagoshima University, Kagoshima 890-0065, \\ Japan \\ ${ }^{3}$ Nobeyama Radio Observatory, National Astronomical Observatory of Japan, Minamimaki, \\ Minamisaku, Nagano 384-1305, Japan \\ ${ }^{4}$ Jodrell Bank Centre for Astrophysics, Alan Turing Building, University of Manchester, \\ Manchester M13 9PL, UK \\ ${ }^{5}$ CSIRO Astronomy and Space Science, PO Box 76, Epping, NSW 1710, Australia
}

\begin{abstract}
A "water fountain" is a transitional object between an AGB star and a PN. The VLBA observations of $22.2 \mathrm{GHz}$ water maser emission reveal a "double-helix" outflow pattern from one of the water fountains, IRAS 18286-0959. The pattern is reasonably fit by a model consisting of two precessing jets. We propose that the two jets observed are a result of a single driving source with a significant proper motion. Using data from the AKARI catalogs, we also found that water fountains might have their own IR colors which are affected by the $9.7 \mu \mathrm{m}$ silicate feature and the optical thickness of stellar envelopes. The colors could serve as new criteria for searching this type of rare objects.
\end{abstract}

Keywords. Circumstellar matter, stars: AGB and post-AGB, stars: individual (IRAS), stars: winds, outflows

\section{Introduction}

Toward the end of stellar evolution, a generally spherical asymptotic giant branch (AGB) star will evolve into a planetary nebula (PN), which usually has a bipolar or multipolar structure (Kwok 2010). A "water fountain (WF)" is a late-AGB or post-AGB star that shows $22.2 \mathrm{GHz} \mathrm{H}_{2} \mathrm{O}$ maser emission with spectral features spreading over a large velocity range (usually $>100 \mathrm{~km} \mathrm{~s}^{-1}$ ), suggesting a high velocity outflow. Such a outflow is closely related to the shaping of a PN (Sahai \& Trauger 1998). IRAS 18286-0959 (I18286) is a member of WFs, which is $\sim 4 \mathrm{kpc}$ apart from us (see contribution by Imai et al., this volume). In this paper, we present the new results of multi-epoch VLBA observations of I18286, and a suggested precession model. The preliminary findings of the AKARI IR color studies on WFs are also discussed.

\section{Results and Discussion}

Figure 1 (left panel) shows the spatial position of each maser feature identified in one of the epochs of the VLBA observations (Epoch C, observed on 2008-Sep-29), together with our double precessing jet model. We found that there are features with different lineof-sight velocities (difference $>50 \mathrm{~km} \mathrm{~s}^{-1}$ ) visually overlapping at both northern and southern ends of the structure in all epochs (Region I and II). The maser distribution as well as the kinematics are best explained by two precessing ballistic jets, jet 1 and jet 2 . The outflow velocity and precessing period of jet 1 are $138 \mathrm{~km} \mathrm{~s}^{-1}$ and 56 years; and for jet 2, they are $99 \mathrm{~km} \mathrm{~s}^{-1}$ and 73 years, respectively (see, Yung et al. 2011, for a detailed 

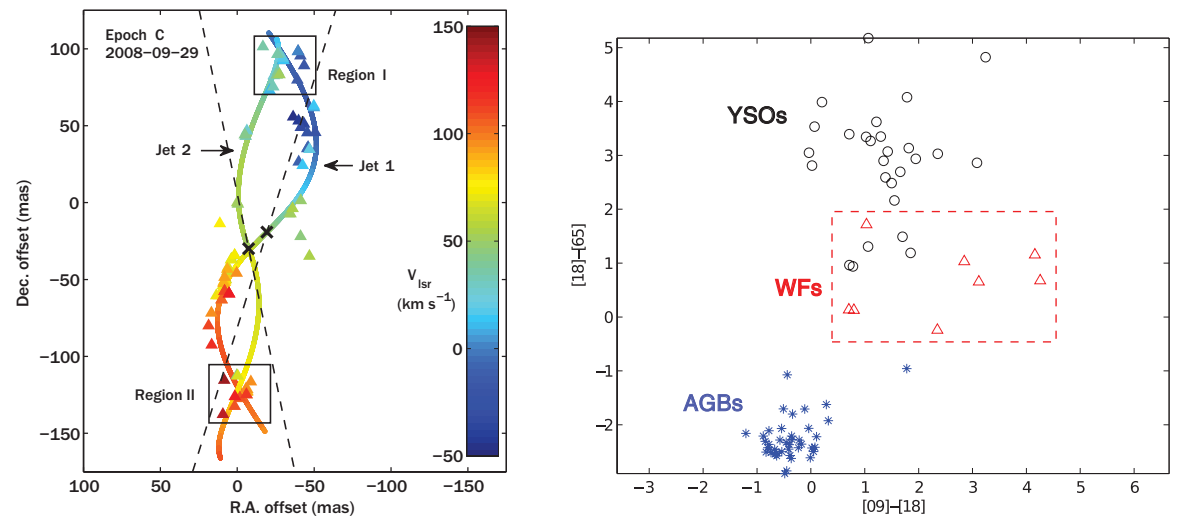

Figure 1. (Left panel) Double precessing jet model for I18286 with two resolvable driving sources. The dotted lines show the projection of the jet axes on the sky plane. The two apparent driving sources are represented by two crosses. (Right panel) Distribution of YSOs (circles), AGBs (asterisks) that have water maser detection, and the water fountains (triangles), on an AKARI color-color diagram. A color version of this figure is available in the electronic version of the Proceedings.

explanation for the model). We propose that the "two" jets are actually generated by one single source but at different instants, and the source itself has a secular motion across the sky, from the position of jet 2's driving source to that of jet 1 . The secular velocity is estimated to be $\sim 27 \mathrm{~km} \mathrm{~s}^{-1}$, if constant.

Figure 1 (right panel) shows the distribution of some YSOs, AGBs (only those with water maser detection) and WFs on an AKARI color-color diagram. There is a clear distinction between the distribution of WFs and that of other AGBs. We believe that the behavior of the $9.7 \mu \mathrm{m}$ silicate feature could be a possible reason causing the distribution difference. As WFs, like I18286, are normally more evolved than normal AGBs, their circumstellar envelopes become optically thicker, and the inner shells are detached from the central stars. The $9.7 \mu \mathrm{m}$ silicate feature will change from an emission feature into an absorption one. In that case, [09]-[18] will become redder as $F_{18} / F_{09}$ increases. On the other hand, the thicker envelope absorbs radiation from the star and re-emits in longer wavelengths. That makes $F_{65} / F_{18}$ and thus [18]- [65] increase, hence the difference observed in both colors axes. If this is confirmed, these AKARI colors could serve as new criteria for selecting WF candidates.

\section{Acknowledgements}

This work is supported by grants from the Research Grants Council of Hong Kong (HKU 703308P, HKU 704209P, HKU 704710P, and HKU 704411P), Small Project Funding of The University of Hong Kong (201007176004), the HKU SPACE Research Fund (20004912), Grant-in-Aid for Young Scientists from the Ministry of Education, Culture, Sports, Science, and Technology (18740109), and Grant-in-Aid for Scientific Research from Japan Society for Promotion Science (20540234). The NRAO is a facility of the National Science Foundation operated under cooperative agreement by Associated Universities, Inc.

\section{References}

Kwok, S. 2010, PASA, 27, 174

Sahai, R. \& Trauger, J. T. 1998, AJ, 116, 1357

Yung, B. H. K., Nakashima, J., Imai, H., Deguchi, S., Diamond, P. J., \& Kwok, S. 2011, ApJ, 741,94 